\title{
Numerical simulation of cavitating flow past axisymmetric body
}

\author{
Dong-Hyun Kim ${ }^{1}$, Warn-Gyu Park ${ }^{1}$ and Chul-Min Jung ${ }^{2}$ \\ ${ }^{1}$ School of Mechanical Engineering, Pusan National University, Busan, Korea \\ ${ }^{2}$ Agency for Defense Development, P.O. Box 18, Jinhae, Kyungnam, Korea
}

\begin{abstract}
Cavitating flow simulation is of practical importance for many engineering systems, such as marine propellers, pump impellers, nozzles, torpedoes, etc. The present work has developed the base code to solve the cavitating flows past the axisymmetric bodies with several forebody shapes. The governing equation is the Navier-Stokes equation based on homogeneous mixture model. The momentum is in the mixture phase while the continuity equation is solved in liquid and vapor phase, separately. The solver employs an implicit preconditioning algorithm in curvilinear coordinates. The computations have been carried out for the cylinders with hemispherical, 1-caliber, and 0-caliber forebody and, then, compared with experiments and other numerical results. Fairly good agreements with experiments and numerical results have been achieved. It has been concluded that the present numerical code has successfully accounted for the cavitating flows past axisymmetric bodies. The present code has also shown the capability to simulate ventilated cavitation.
\end{abstract}

KEY WORDS: Cavitating flow; Navier-stokes equations; Homogeneous mixture model; Axisymmetric bodies; Reentrant jet; Ventilated cavitation. NOMENCLATURE

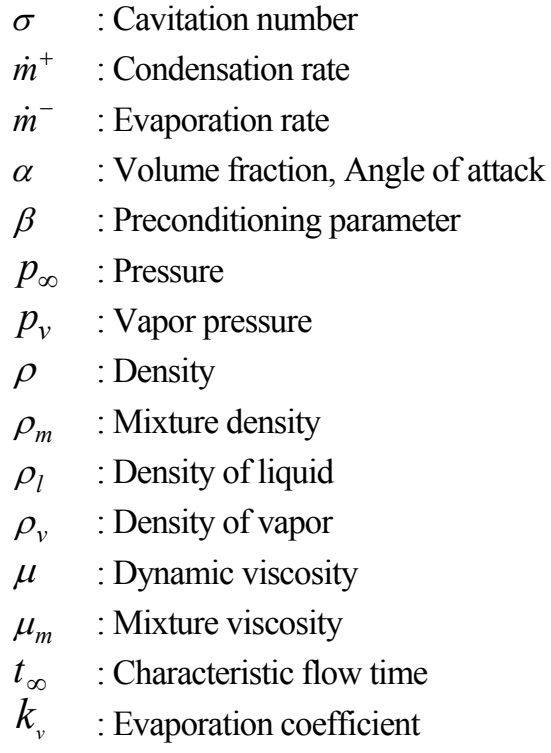

$\begin{array}{ll}\sigma & : \text { Cavitation number } \\ \dot{m}^{+} & : \text {Condensation rate } \\ \dot{m}^{-} & : \text {Evaporation rate } \\ \alpha & : \text { Volume fraction, Angle of attack } \\ \beta & : \text { Preconditioning parameter } \\ p_{\infty} & : \text { Pressure } \\ p_{v} & : \text { Vapor pressure } \\ \rho & : \text { Density } \\ \rho_{m} & : \text { Mixture density } \\ \rho_{l} & : \text { Density of liquid } \\ \rho_{v} & : \text { Density of vapor } \\ \mu & : \text { Dynamic viscosity } \\ \mu_{m} & : \text { Mixture viscosity } \\ t_{\infty} & : \text { Characteristic flow time } \\ k_{v} & : \text { Evaporation coefficient }\end{array}$

$k_{l} \quad$ : Condensation coefficient

$k_{p} \quad$ : Scaling coefficient

$\tau \quad$ : Pseudo time

$\tau_{\text {ref }}:$ Reference time

$\tau_{\text {relax }}:$ Relaxation time

$\hat{S} \quad$ : Source vector

Re : Reynolds number

$V \quad$ : Velocity

$H \quad$ : Water depth

$\Gamma \quad$ : Precondition matrix

$\Gamma_{\mathrm{e}} \quad$ : Flux jacobian matrix

$u, v, w \quad$ : Cartesian velocity

$\widehat{E}, \hat{F}, \widehat{G} \quad$ : Flux vector

$\widehat{E}_{v}, \widehat{F}_{v}, \widehat{G}_{v}:$ Solution vector

Corresponding author: Warn-Gyu Park

e-mail:wgpark@pusan.ac.kr 


\section{INTRODUCTION}

Cavitation generally occurs if the pressure in a certain region of liquid flow drops below the vapor pressure and, consequently, the liquid is vaporized and filled with cavity. The cavitating flow is usually observed in various propulsion systems and high-speed underwater objects, such as marine propellers, impellers of turbomachinery, hydrofoils, nozzles, torpedoes, etc. This phenomenon usually causes severe noise, vibration and erosion.

Even though cavitating flow is a complex phenomenon which has not been completely modeled, a lot of attention was gathered in CFD community as the methodologies for single-phase flow was relatively much matured. In solving multiphase flows by CFD method, they can be categorized into three groups: The first group use a single continuity equation (Reboud and Delannoy, 1994; Song and He, 1984). This method has been known that it is unable to distinguish between condensable and non-condensable vapor (Kunz, Lindau, Billet and Stinebring, 2001). Next group is to solve separate continuity equations for liquid and vapor phases by adding source terms accounting for the mass transfer between phases (Kunz, Lindau, Billet and Stinebring, 2001; Merkle, Feng and Buelow, 1998; Kunz, et al., 2000; Ahuja, Hosangadi and Arunajatesan, 2001; Shin and Itohagi, 1998). This model is usually so called 'homogeneous mixture model', because the liquid-gas interface is assumed to be in dynamically and thermally equilibrium in the process of mass transfer between liquid and vapor phases and, consequently, mixture phase of momentum and energy equations are used. They consider in the process of the mass transfer between liquid and vapor phases. Final group solves full two-fluid modeling, wherein separate momentum and energy equations are employed for the liquid and the vapor phase (Grogger and Alajbegovic, 1998; Staedtke, Deconinck and Romenski, 2005). This method is widely used in nuclear engineering.

If adding more information to the second group that uses the homogeneous mixture model, some authors have reported preconditioning algorithms for multiphase mixtures. Kunz, et al. (2000) developed a code for the presence of a non-condensable vapor. The governing equations, in which a separate continuity equation is used for an individual phase while the momentum equations are described for the mixture phase, were solved by using the preconditioning and the dual time stepping method. However, in this model, the compressibility effects were not taken into account in the multiphase mixture region. Recently, Lindau, Venkateswaran, Kunz and Merkle (2003) and Owis and Nayfeh (2003) have been presented the fully compressible multiphase flow models which have taken into account the changes of both compressibility and temperature.

Coutier-Delgosha, Patella and Reboud (2003) reported that the unsteadiness of cavitating flows strongly depends on the turbulence model, and it has a great effect on the mean and fluctuating fields of vapor fraction and velocity. A simple modification of turbulence model was introduced to reduce the effective viscosity in the mixture and to take into account the influence of liquid-vapor mixture high compressibility on the turbulence structure.

Senocak and Shyy (2004) compared three cavitation models, namely by Merkle, Feng and Buelow (1998), Kunz, et al. (1999), and Singhal, Athavale, Li and Jiang (2002). The comparison of surface pressure distribution over a hemispherical object gave a good agreement among these three models. However, the density profiles do not reach an agreement each other, indicating that the cavitation models generate different compressibility characteristics. A new interfacial dynamics-based cavitation model was also developed for steady flow case, resulting in an additional equation for the normal velocity of the vapor phase on the interface.

The objective of the present work is to develop an in-house base code which will be used to simulate cavitating flow past supercavitating projectiles, whose final goal is to include the effects of condensable/non-condensable vapor, compressibility effects, and hot plume gas of propulsive exhaust gas after long-term nine years project. The present goal of the first stage for three years is to develop the base code that would follow the homogeneous mixture models, and then compare the results with other published numerical and experimental results.

\section{MAIN TEXT}

\section{Governing equations and numerical method}

Based on the homogeneous mixture model, the governing equation is comprised of the continuity equation for liquid and vapor phases and momentum equation in mixture phase as follows (2000): 


$$
\begin{gathered}
\left(\frac{1}{\rho_{m} \beta^{2}}\right) \frac{\partial p}{\partial \tau}+\frac{\partial u_{j}}{\partial x_{j}}=\left(\dot{m}^{+}+\dot{m}^{-}\right)\left(\frac{1}{\rho_{l}}-\frac{1}{\rho_{v}}\right) \\
\frac{\partial \alpha_{l}}{\partial t}+\left(\frac{\alpha_{l}}{\rho_{m} \beta^{2}}\right) \frac{\partial p}{\partial \tau}+\frac{\partial \alpha_{l}}{\partial \tau}+\frac{\partial}{\partial x_{j}}\left(\alpha_{l} u_{j}\right)=\left(\dot{m}^{+}+\dot{m}^{-}\right)\left(\frac{1}{\rho_{l}}\right) \\
\frac{\partial}{\partial t}\left(\rho_{m} u_{i}\right)+\frac{\partial}{\partial \tau}\left(\rho_{m} u_{i}\right)+\frac{\partial}{\partial x_{j}}\left(\rho_{m} u_{i} u_{j}\right)=-\frac{\partial p}{\partial x_{j}}+\frac{\partial}{\partial x_{j}}\left[\mu_{m, t}\left(\frac{\partial u_{i}}{\partial x_{j}}+\frac{\partial u_{j}}{\partial x_{i}}\right)\right]
\end{gathered}
$$

where the subscript $l$ and $v$ mean the liquid and vapor phase, respectively. The subscript $m$ denotes the mixture phase. $\alpha$ is the volume fraction. $\tau$ is the pseudo-time for dual time stepping while $t$ is the physical time. $\dot{m}^{+}$and $\dot{m}^{-}$denote transformation of vapor to liquid and that of liquid to vapor, respectively. The density of the mixture phase is defined as:

$$
\begin{gathered}
\rho_{m}=\alpha_{l} \rho_{l}+\alpha_{v} \rho_{v} \\
\alpha_{l}+\alpha_{v}=1
\end{gathered}
$$

The molecular viscosity, $\mu_{m}$, is computed as

$$
\mu_{m}=\alpha_{l} \mu_{l}+\alpha_{v} \mu_{v}
$$

Turbulent eddy viscosity, $\mu_{t}$, is obtained from Chien $k$ - $\varepsilon$ model (1982) by using

$$
\mu_{t}=\frac{\rho_{m} c_{\mu} f_{\mu} k^{2}}{\varepsilon}
$$

where $k$ is the turbulent kinetic energy and $\varepsilon$ is the turbulent dissipation rate. $c_{\mu}$ is empirical constants and $f_{\mu}$ is damping function.

Rewriting Eqs. (1) in generalized curvilinear coordinates,

$$
\Gamma_{e} \frac{\partial \hat{q}}{\partial t}+\Gamma \frac{\partial \hat{q}}{\partial \tau}+\frac{\partial\left(\hat{E}-\hat{E}_{v}\right)}{\partial \xi}+\frac{\partial\left(\hat{F}-\hat{F}_{v}\right)}{\partial \eta}+\frac{\partial\left(\hat{G}-\hat{G}_{v}\right)}{\partial \zeta}=\hat{S}
$$

where $\hat{q}=J^{-1}\left[\begin{array}{lllll}p & u & v & w & \alpha_{l}\end{array}\right]^{T} . \hat{E}, \hat{F}$, and $\hat{G}$ denote convective flux along $\xi$, $\eta$-, $\zeta$-direction, respectively. $\hat{E}_{v}, \hat{F}_{v}$, and $\hat{G}_{v}$ denote viscous flux terms along each direction. The flux Jacobian matrix, $\Gamma_{e}$ and $\hat{S}$ are represented as:

$$
\Gamma_{e}=\left[\begin{array}{ccccc}
0 & 0 & 0 & 0 & 0 \\
0 & \rho_{m} & 0 & 0 & u \Delta \rho_{1} \\
0 & 0 & \rho_{m} & 0 & v \Delta \rho_{1} \\
0 & 0 & 0 & \rho_{m} & w \Delta \rho_{1} \\
0 & 0 & 0 & 0 & 1
\end{array}\right]
$$




$$
\hat{S}=J\left\{\left(\dot{m}^{+}+\dot{m}^{-}\right)\left(\frac{1}{\rho_{l}}-\frac{1}{\rho_{v}}\right), \quad 0, \quad 0, \quad 0, \quad\left(\dot{m}^{+}+\dot{m}^{-}\right) \frac{1}{\rho_{l}}\right\}^{T}
$$

where $\Delta \rho_{l} \equiv \rho_{l}-\rho_{v}$. The pre-conditioning matrix, $\Gamma$, is obtained from the modification of $\Gamma_{e}$ to efficiently handle the stiffness problem that comes from significant difference of the speed of sound in liquid and vapor phase (2000) as:

$$
\Gamma=\left[\begin{array}{ccccc}
\frac{1}{\rho_{m} \beta^{2}} & 0 & 0 & 0 & 0 \\
0 & \rho_{m} & 0 & 0 & u \Delta \rho_{1} \\
0 & 0 & \rho_{m} & 0 & v \Delta \rho_{1} \\
0 & 0 & 0 & \rho_{m} & w \Delta \rho_{1} \\
\frac{\alpha_{l}}{\rho_{m} \beta^{2}} & 0 & 0 & 0 & 1
\end{array}\right]
$$

where $\beta$ is the preconditioning parameter.

The numerical methodology for solving Eq. (6) has used the iterative time marching method by Park and Sankar (1993) and Park, Jang, Chun and Kim (2005), that was developed for unsteady incompressible Navier-Stokes equations with artificial compressibility method of Rogers and Kwak (1991).

\section{Cavitation modeling}

Cavitation model, based on Lindau, Venkateswaran, Kunz and Merkle (2003), was used in the present work. In this model, the mass transfer rate from liquid to vapor in a region where the local pressure is less than the vapor pressure is simply modeled as being proportional to the liquid volume fraction and the difference between the local and vapor pressures, as follows:

$$
\dot{m}^{-}=\frac{C_{d e s t} \rho_{v} \alpha_{l} \min \left[0, p-p_{v}\right]}{\left(\rho_{l} U_{\infty}^{2} / 2\right) t_{\infty}}
$$

where $p_{v}$ denotes vapor pressure of the liquid. The mass transfer from vapor back to liquid in a region where the local pressure exceeds the vapor pressure is given as follows:

$$
\dot{m}^{+}=\frac{C_{p r o d} \rho_{v} \alpha_{l}^{2}\left(1-\alpha_{l}\right)}{t_{\infty}}
$$

In the present work, the empirical coefficients, $C_{\text {dest }}$ and $C_{\text {prod }}$, was set as: $C_{\text {dest }}=10^{5}, C_{\text {prod }}=200$. Both mass transfer rates are non-dimensionalized with respect to a mean flow time scale.

\section{Results and discussion}

The present code was applied to the cavitating flows past the axisymmetric bodies with hemispherical, 1-caliber, and 0caliber forebody shape to compare with other numerical and experimental results. Fig.1 shows the grid for the hemispherical forebody consisting of $120 \times 137 \times 37$ grid points. The computations were carried out for four cavitation numbers from $\sigma=0.2$ to 0.5 at $R_{e}=1.36 \times 10^{5}$. The cavitation number, $\sigma$, is defined as:

$$
\sigma=\frac{p_{\infty}-p_{v}}{0.5 \rho_{\infty} U_{\infty}^{2}}
$$




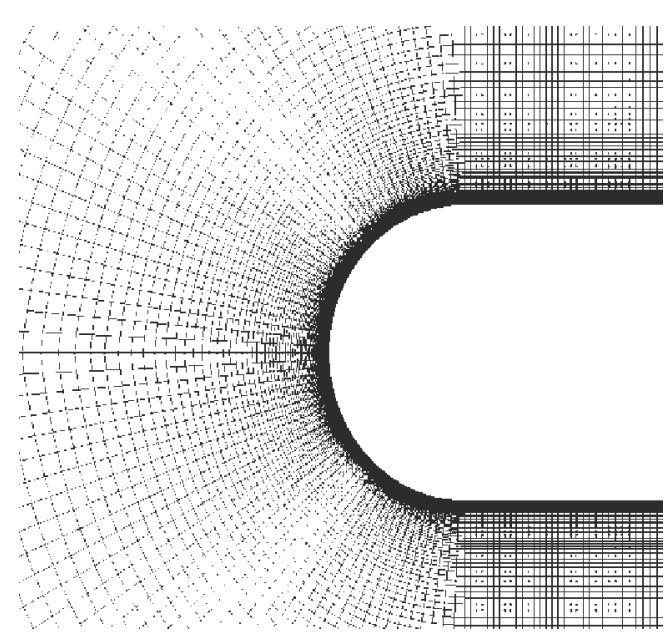

Fig. 1 Grid of hemispherical forebody body.

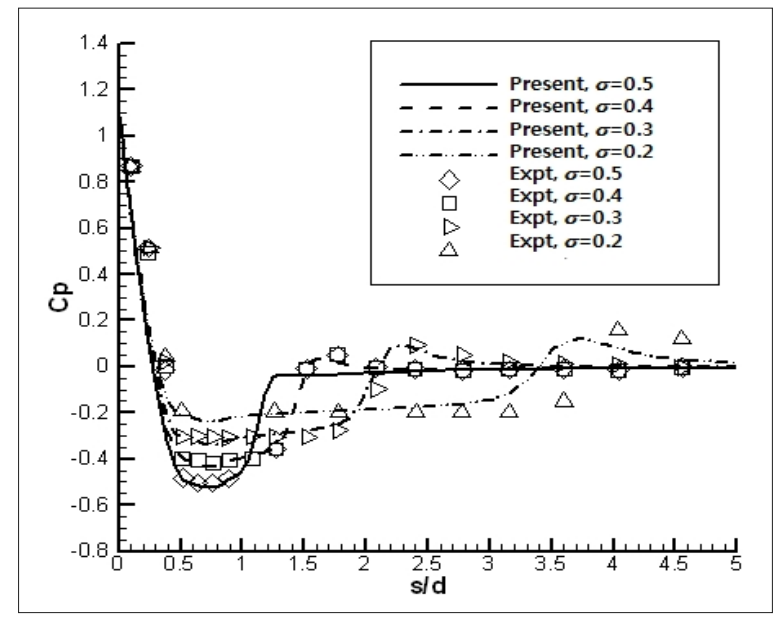

Fig. 2 Surface pressure coefficient on the hemispherical cylinder.

Fig. 2 shows surface pressure coefficient, compared with experiment of Rouse and McNown (1948). A fairly good agreement with experiment was obtained, except in the cavity closure region at $\sigma=0.2$. The cavity size at $\sigma=0.2$ was a little underpredicted than that of experiment. This discrepancy may be attributed to the limitation of k- $\varepsilon$ turbulence model and the grid resolution in the closure region. Stutz and Reboud (1997) noted that the vorticity production, especially in the closure region, is the important factor for computing cavitating flows. The k- $\varepsilon$ turbulence model and cavitation model used in the present work may not account for the vorticity production in the closure region. These limitations may be pronounced by the large cavity length at $\sigma=0.2$. Fig. 3 shows the comparison of liquid volume fractions with numerical result by Kunz, et al. (1999) at $\sigma=0.3$, indicating that the cavity inception points, total cavity size and flow features with reentrant jet agree well each other.

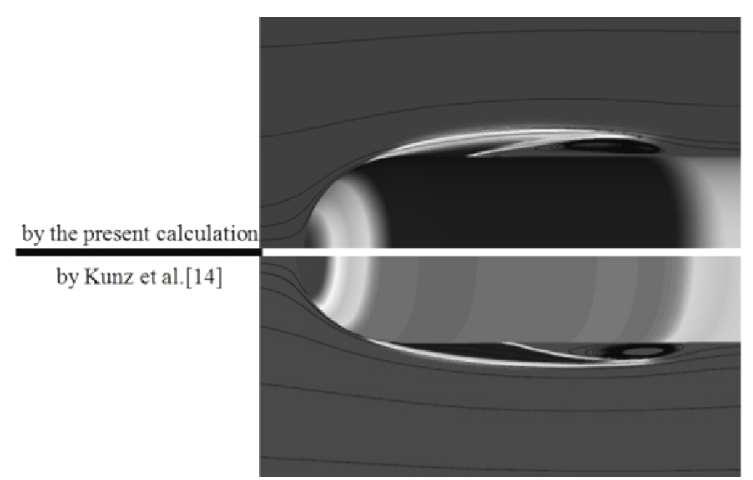

Fig. 3 Comparison with other numerical result at $\sigma=0.3$.

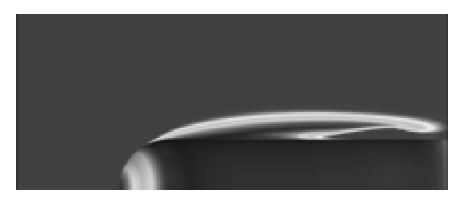

(a) $\sigma=0.2$.

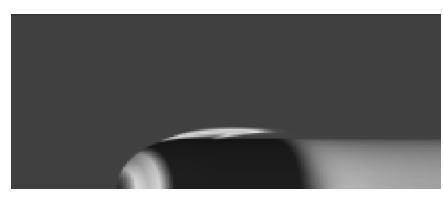

(c) $\sigma=0.4$.

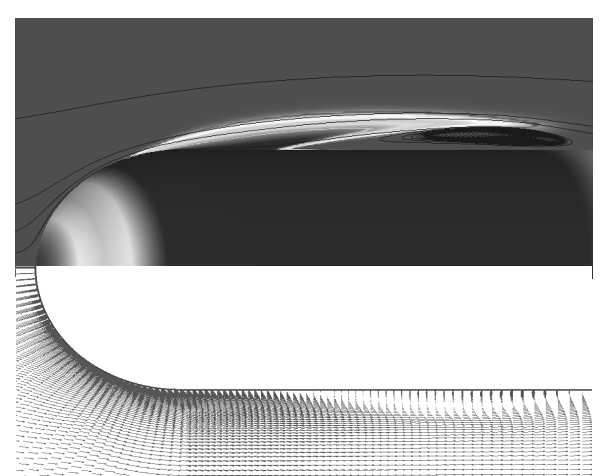

Fig. 4 Velocity vectors, streamlines, surface pressure distribution, and liquid volume fraction of hemispherical cylinder at $\sigma=0.3$.

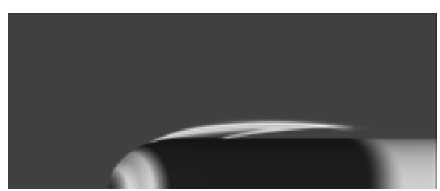

(b) $\sigma=0.3$.

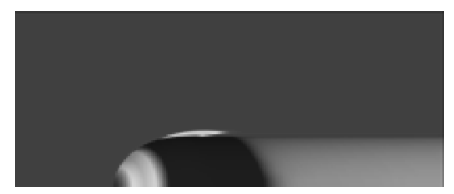

(d) $\sigma=0.5$.

Fig. 5 Liquid volume fraction and surface pressure contour of hemispherical cylinder at different cavitation numbers. 


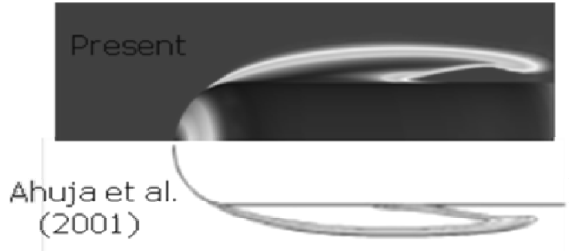

(a) $\sigma=0.2$.

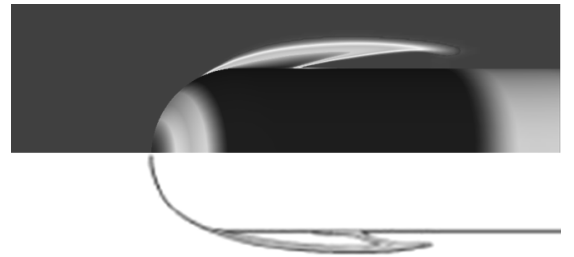

(b) $\sigma=0.3$.

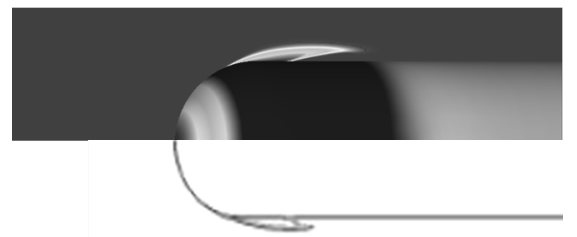

(c) $\sigma=0.4$.

Fig. 6 Comparison of liquid volume fraction with other numerical result (Ahuja, Hosangadi and Arunajatesan, 2001).

Fig. 4 shows velocity vectors together with streamlines and liquid volume fraction. Fig. 5 shows liquid volume fraction and surface pressure contour at different cavitation numbers and also shows the cavity length is reduced as the cavitation number increases, as expected. Fig. 6 shows the liquid volume fraction at three different cavitation numbers, compared with numerical result by Ahuja, Hosangadi and Arunajatesan (2001). This figure also shows the present result is in a good accordance with the results by other researcher.

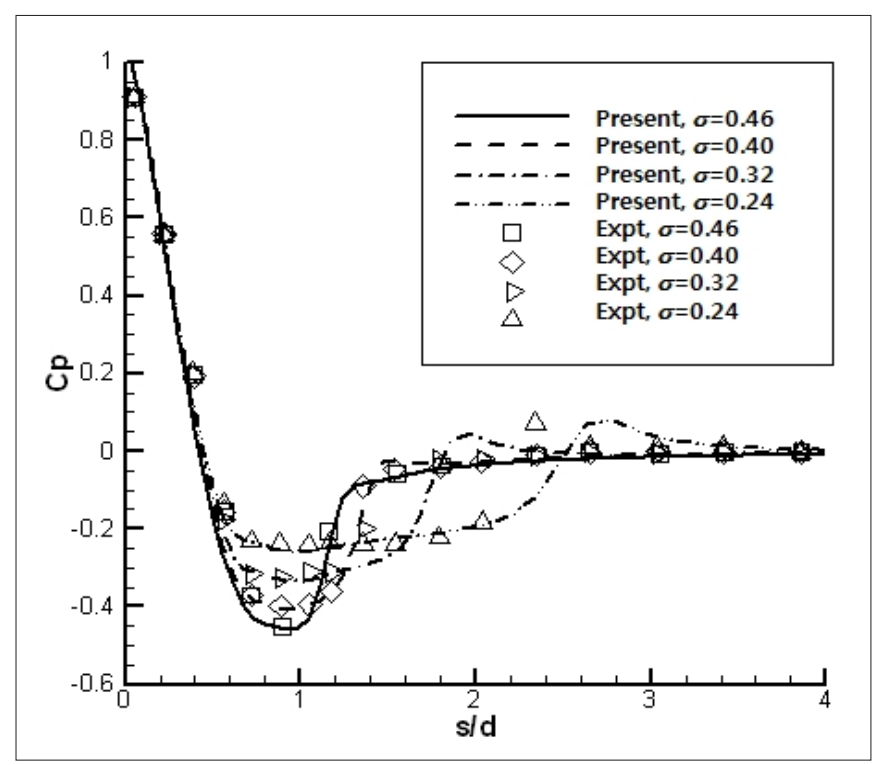

Fig. 7 Surface pressure coefficient on the 1-caliber ogive cylinder.

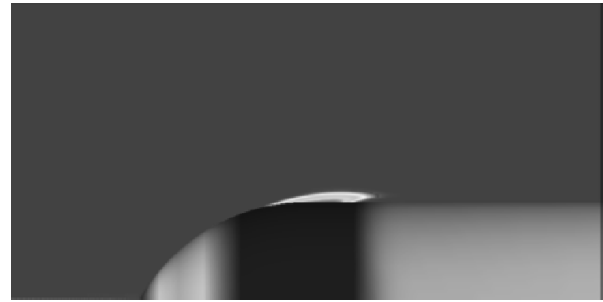

(a) $\sigma=0.32$

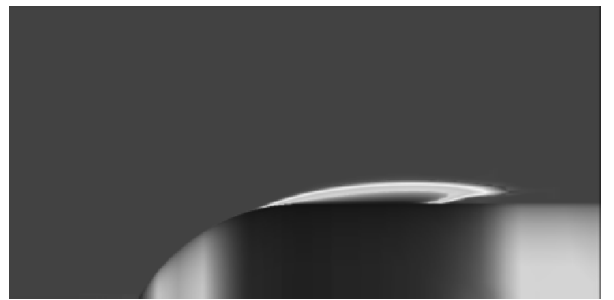

(b) $\sigma=0.24$.

Fig. 8 Liquid volume fraction and surface pressure distribution of 1-caliber cylinder at two cavitation numbers. 
Fig. 7 shows surface pressure distribution on the 1-caliber cylinder at four different cavitation numbers, compared with experiments (Rouse and McNown,1948). Fig. 8 shows the liquid volume fraction and surface pressure distribution at $\sigma=0.32$ and 0.24 .

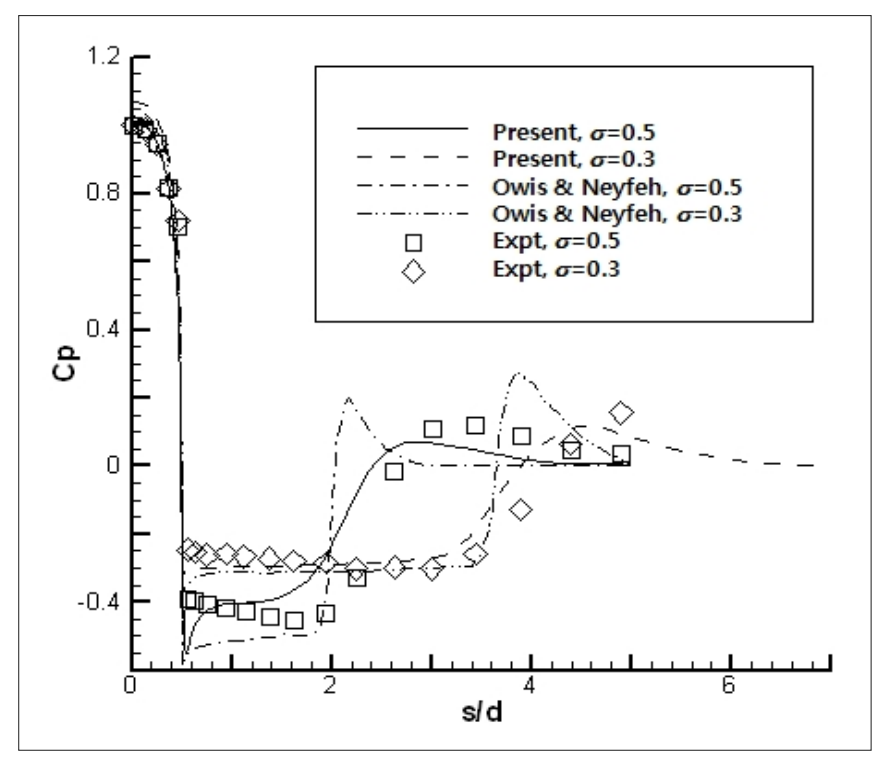

Fig. 9 Surface pressure coefficient on the 0-caliber cylinder.

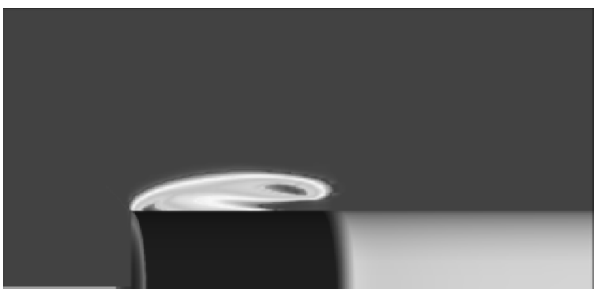

(a) $\sigma=0.5$.

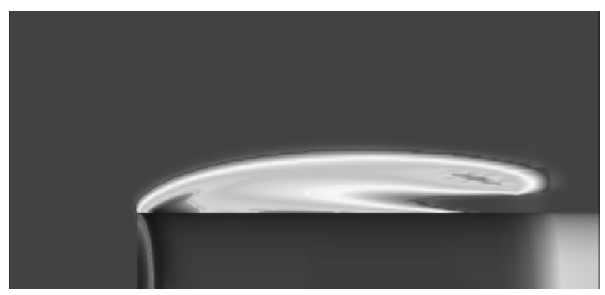

(b) $\sigma=0.3$.

Fig. 10 Liquid volume fraction of 0 -caliber cylinder at two cavitation numbers.

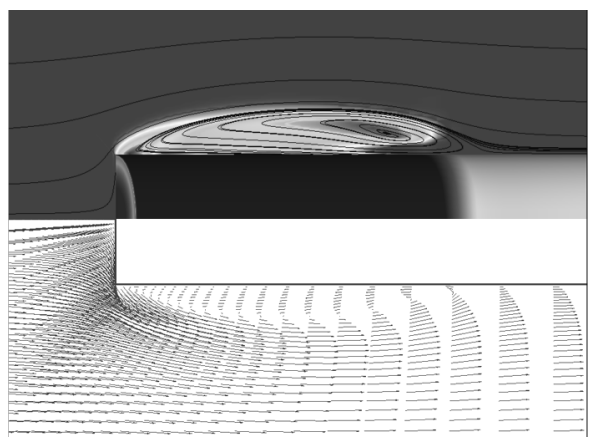

(a) Velocity vectors, streamlines, and liquid volume fraction.

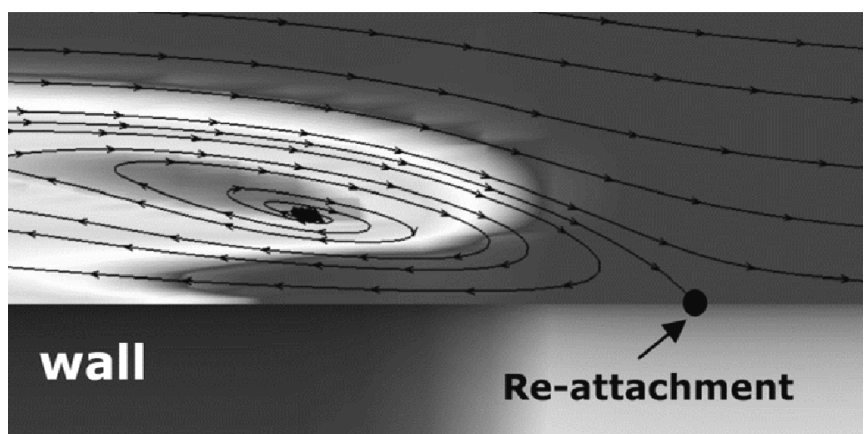

(b) Re-attachment point in cavity closure region.

Fig. 11 Cavitating flow past 0 -caliber cylinder at $\sigma=0.3$.

Fig. 9 shows the surface pressure coefficient on the 0 -caliber cylinder. The results were compared with numerical results by Owis and Nayfeh (2003) and experiments (Rouse and McNown, 1948). Fig. 10 shows liquid volume fraction at $\sigma=0.5$ and 0.3. Fig. 11 shows flow features of 0 -caliber cylinder at $\sigma=0.3$. In Fig. 11(b), it is shown that detached flow by the cavity is reattachment point at cavity closure. High pressure at the re-attachment region was shown in the plot of surface pressure coefficients of Fig. 2, 7, and 9. This high pressure at the re-attachment region plays a major role in the formation of the re-entrant 
jet phenomenon. The detail flow pattern by the re-entrant jet is shown in Fig. 14

Fig. 12 shows another comparison with the result of Lindau, Kunz, Venkateswaran and Stinebring (2004). If viewing animation, both of results, by the present and Kunz et al., have shown that the cavity rotates in the clockwise direction. Fig. 13 shows the comparison of the liquid volume fraction and streamlines with other numerical result (Venkateswaran, Lindau, Kunz and Merkle, 2001). This figure shows comparatively good agreement. Fig. 14 shows the flow pattern of the re-entrant jet. The high pressure near the re-attachment region induces strong re-entrant jet, flowing reversely into the cavity. This re-entrant jet lifts the cavity off as shown in figure at $t=0.4$ and, then, cuts the cavity as shown in figures at $t=0.8$ and 1.2. The periodic pattern of this phenomena restarts at figure at $t=2.0$. This periodic flow pattern induces unsteadiness and the time history of drag coefficient is shown in Fig.15. The period is about $t=2$, which is consistent with that of Venkateswaran, Lindau, Kunz and Merkle (2001). Fig. 16 shows the comparison of the cavity size without and with condensable vapor blowing at the shoulder of the nose. The condensable vapor $\left(\sigma_{v}=1.0\right)$ was assumed to be blown with $p_{b}=2 p_{\infty}, V_{b}=2 V_{\infty}$, and $T_{b}=T_{\infty}$. The subscript ' $b$ ' denotes the blowing at the nose shoulder circumferentially at the location of Fig. 16(a). The resulting cavities with condensable vapor blowing show directly comparison of without and with vapor blowing at same vapor volume fraction contour. The vapor blowing technique, so called 'ventilated cavitation', plays a major role in supercavitating torpedo to drastically reduce the drag. Fig. 15 shows that the present code has some capability toward the ventilated cavitation for the supercavitating torpedo. This capability is the first success over the other researchers who stayed in non-ventilated cavitating flows. The present code will be further improved to analyze the natural and ventilated non-condensable vapor cavitation of the supercavitation vehicle.

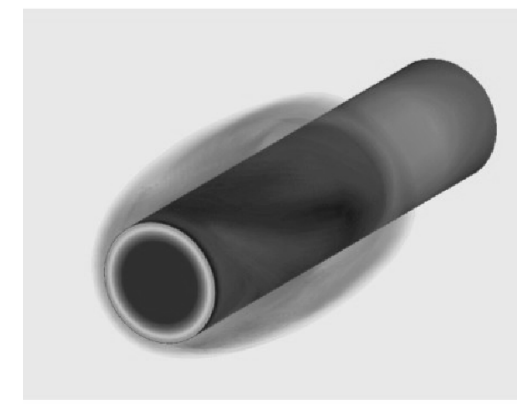

(a) Present result.

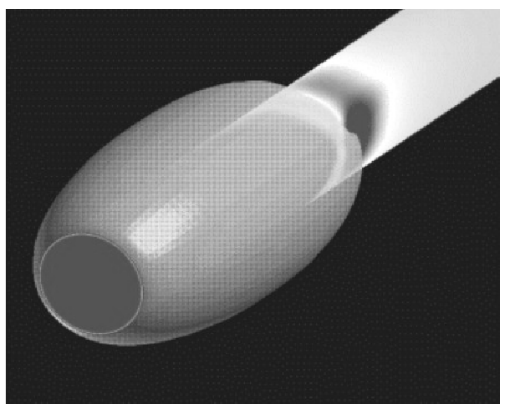

(b) Result by Lindau, Kunz, Venkateswaran and Stinebring (2004).

Fig. 12 Comparison with liquid volume fraction of 0 -caliber cylinder at $\sigma=0.3$.
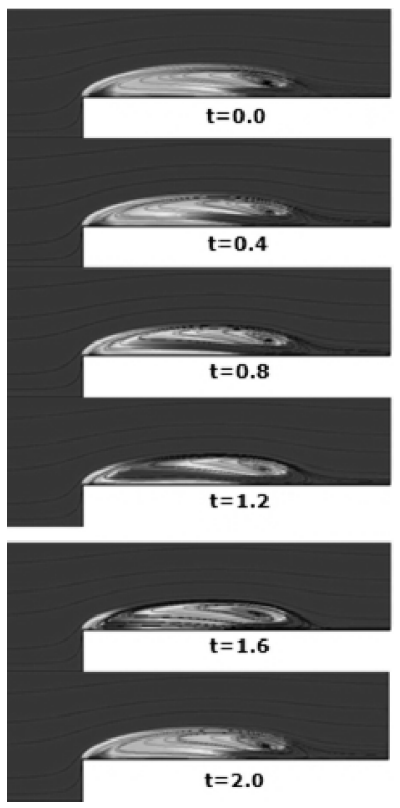

(a) Present.
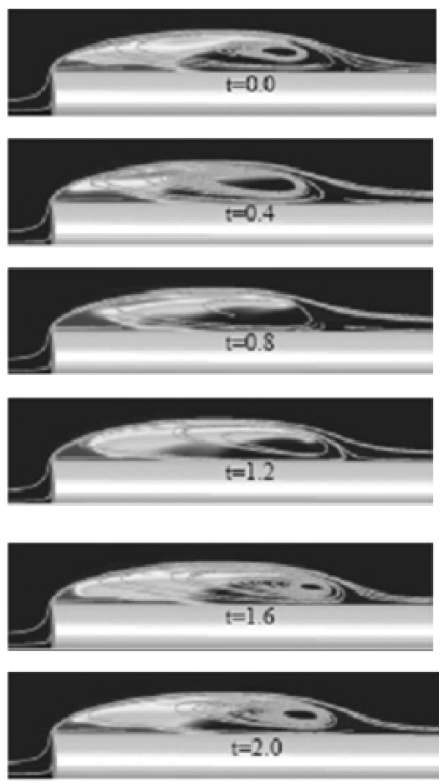

(b) Venkateswaran, Lindau, Kunz and Merkle (2001).

Fig. 13 Comparison of the liquid-volume-fraction and streamlines of 0-caliber cylinder at $\sigma=0.3$. 


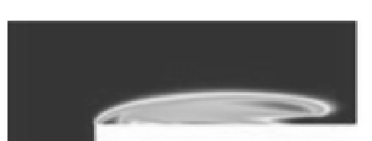

(a) $\mathrm{t}=0.0$

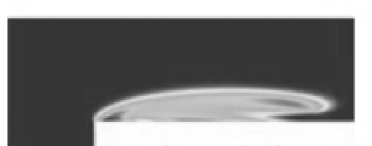

(b) $\mathrm{t}=0.13$

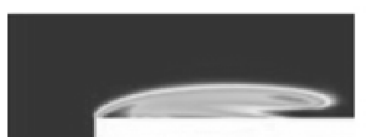

(c) $\mathrm{t}=0.26$

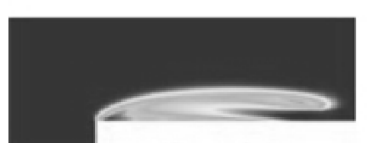

(d) $\mathrm{t}=0.4$

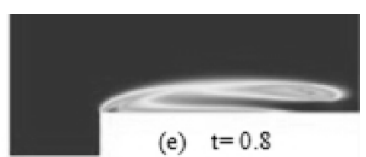

(e) $\mathrm{t}=0.8$

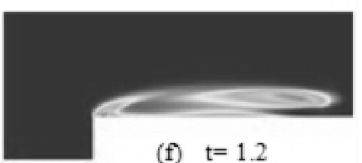

(f) $\mathrm{t}=1.2$

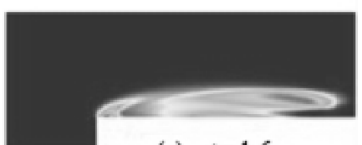

(g) $\mathrm{t}=1.6$

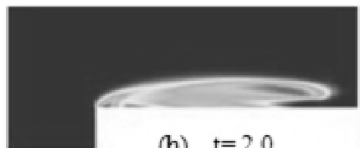

(h) $\mathrm{t}=2.0$

Fig. 14 Flow pattern by re-entrant jet.

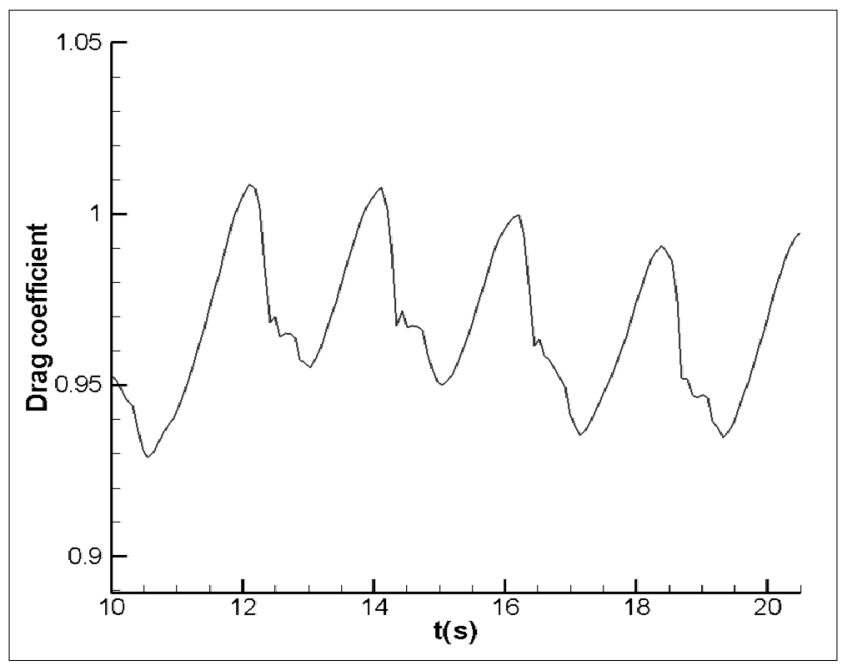

Fig. 15 Time history of drag coefficient past 0 -caliber cylinder at $\sigma=0.3$.

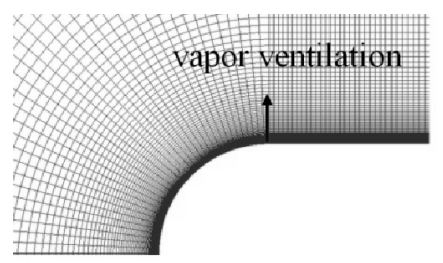

(a) Location of ventilation.

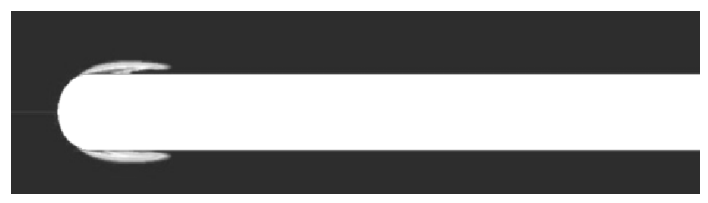

(b) Without gas blowing.

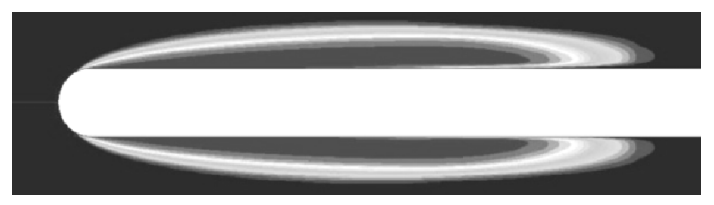

(c) With gas blowing.

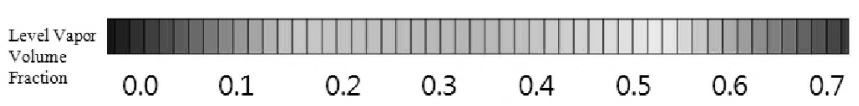

Fig. 16 Cavity size with and without condensable vapor blowing. 


\section{CONCLUSIONS}

The base code for simulating the cavitating flow around the supercavitation vehicle has been developed. The present code that used the homogeneous mixture method solved the cavitating flows past hemispherical, 1-caliber, and 0-caliber forebody cylinders at various cavitation numbers. The results by the present code have shown a good agreement with experiments and other numerical results. Hence, it was concluded that the present code could have the capacity as the base code toward the cavitating flow analysis code for the supercavitation vehicle, having natural and ventilated cavitation, condensable and/or noncondensable vapor blowing, and hot exhaust vapor of propulsive rocket. The present result has shown the strong unsteady phenomena of the re-entrant jet. Also, it has shown the capability of the ventilated cavitation.

\section{ACKNOWLEDGEMENTS}

This work was supported by the Human Resources Development of the Korea Institute of Energy Technology Evaluation and Planning (KETEP) grant funded by the Korea government Ministry of Knowledge Economy (No. 20114010203080) and Underwater Vehicle Research Center (UVRC).

\section{REFERENCES}

Ahuja, V., Hosangadi, A. and Arunajatesan, S., 2001. Simulation of cavitating flow using hybrid unstructured meshes. Journal of Fluids Engineering, 123, pp.331-340.

Chien, K. Y., 1982. Prediction of change and boundary layer flows with a low-Reynolds-number turbulence model. $A I A A$ Journal, 20(1), pp.33-38.

Coutier-Delgosha, O., Patella, R. F. and Reboud, J.L., 2003. Evaluation of the turbulence model influence on the numerical simulations of unsteady cavitation. Journal of Fluids Engineering, 125(1), pp.38-45.

Grogger, H. A. and Alajbegovic, A., 1998. Calculation of the cavitating flow in venturi geometries using two fluid model. ASME Paper FEDSM 98-5295.

Kunz, R. F., Lindau, J. W., Billet, M. L. and Stinebring, D. R., 2001. Multiphase CFD modeling of developed and supercavitaing flows. Proceedings of the Von Karman Institute, special course on supercavitating flows. Rhode-Saint-Genese, Belgium, pp.12-16.

Kunz, R. F., Boger, D. A., Stinebring, D. R., Chyczewski, T. S., Lindau, J. W., Gibeling, H. J., Venkateswaran, S. and Govindan, T. R., 2000. A preconditioned Navier-Stokes method for two-phase flows with application to cavitation prediction. Computers \& Fluids, 29(8), pp.849-875.

Kunz, R. F., Boger, D. A., Chyczewski, T. S., Stinebring D. R., Gibeling, H. J. and Govindan, T. R., 1999. Multi-phase CFD analysis of natural and ventilated cavitation about submerged bodies. ASME Paper FEDSM 99-7364.

Lindau, J. W., Venkateswaran, S., Kunz, R. F. and Merkle, C. L, 2003. Computation of compressible multiphase flows. AIAA Paper 2003-1285.

Lindau, J. W., Kunz, L .F., Venkateswaran, S. and Stinebring, D. R., 2004. Homogeneous multiphase CFD modeling of large scale cavities. ECCOMAS 2004. Jyväskylä, Finland.

Merkle, C. L., Feng, J. Z. and Buelow, P. E. O., 1998. Computational modeling of the dynamics of sheet cavitation. Proceedings of the $3^{\text {rd }}$ international symposium on cavitation. Grenoble, France, pp.307-311.

Owis, F. M. and Nayfeh, A. H., 2003. Computations of the compressible multiphase flow over the cavitating high-speed torpedo. Journal of Fluids Engineering, 125(3), pp.459-468.

Park, W-G. and Sankar, L. N., 1993. A technique for the prediction of unsteady incompressible viscous flows. AIAA Paper 93-3006.

Park, W-G., Jang, J. H., Chun, H. H. and Kim, M. C., 2005. Numerical flow and performance analysis of waterjet propulsion system. Ocean Engineering, 32(14-15), pp.1740-1761.

Reboud, J. L. and Delannoy, Y., 1994. Two-phase flow modeling of unsteady cavitation. Proceedings of $2^{\text {nd }}$ international symposium on cavitation. Tokyo, Japan, pp.39-44.

Rogers, S.E. and Kwak, D., 1991. Steady and unsteady solutions of the incompressible Navier-Stokes equations. AIAA JOurnal, 29(4), pp.603-610. 
Rouse, H. and McNown, J.S., 1948. Cavitation and pressure distribution, head forms at zero angle of yaw. Studies in engineering. Bulletin, 32, State University of Iowa.

Song, C. and He, J., 1984. Numerical simulation of cavitating flows by single-phase flow approach. Proceedings of $3^{\text {rd }}$ international symposium on cavitation. Grenoble, France, pp.295-300.

Shin, B. R. and Itohagi, T., 1998. A numerical study of unsteady cavitating flows. Proceedings of the $3^{\text {rd }}$ international symposium on cavitation. Grenoble, France, pp.301-306.

Staedtke, H., Deconinck, H. and Romenski, E., 2005. Advanced three-dimensional two-phase flow simulation tools for application reactor safety(ASTAR). Nuclear Engineering and Design, 235(2-4), pp.379-400.

Senocak, I. and Shyy, W., 2004. Interfacial dynamics-based modeling of turbulent cavitating flows, Part-2: Time-dependent computations. International Journal for Numerical Methods in Fluids, 44(9), pp.997-1016.

Singhal, A. K., Athavale, M. M., Li, H. and Jiang, Y., 2002. Mathematical basis and validation of the full cavitation model. Journal of Fluids Engineering, 124(3), pp.617-624.

Stutz, B. and Reboud, J-L., 1997. Two-phase flow structure of sheet cavitation. Physics of Fluids, 9(12), pp.3678-3686.

Venkateswaran, S., Lindau, J. W., Kunz, R.F. and Merkle, C. L., 2001. Preconditioning algorithms for the computation of multi-phase mixture flows. AIAA Paper 2001-0279. 